\title{
CULTURAS ACADÉMICAS: ENTRE REINVENCIÓN Y CONTRABANDO
}

\section{Academic cultures between reinvention and smuggling}

\author{
Kathya Araujo* \\ * Universidad Academia de Humanismo Cristiano (Chile) \\ karaujo@academia.cl
}

\begin{abstract}
Palabras clave
Culturas

académicas

locales

Evaluación

Rol crítico y

público

Migración y chilena), y la reinvención y el contrabando que toda migración supone, este artículo argumenta de qué manera frente a las nuevas modalidades de evaluación (los rankings, la búsqueda de isomorfismo de las estructuras argumentativas y las formas lingüísticas o incluso la ficción de sociologías globales), se mantienen vivas culturas académicas particulares. En este marco, la reflexión se organiza mostrando que detrás de la singularidad de los estilos intelectuales, formas de sociabilidad profesionales e incluso formas lingüísticas de cada una de ellas, lo que se revela es la manera en la que cada cultura académica local responde desde tradiciones diversas a desafíos político sociales distintos y con ello su rol de compromiso público y crítico.
\end{abstract}

\section{Resumen}

Sobre la base del análisis de una historia personal de desplazamiento entre academias (peruana

\section{Abstract}

Based upon the analysis of a personal trajectory which implied shifting between Peruvian and Chilean academic milieus, this paper argues how in despite of new evaluations modalities (rankings, the search for isomorphic structures of argumentation and linguistic forms or even

Keywords

Local academic cultures

Evaluation

Public and critical role

Migration the fiction of global sociologies), particular academic cultures stay alive. In this regard, it intends to show that the singularity of intellectual styles, forms of professional sociability or even of linguistic and rhetoric uses in each one of them are nothing else but the manner in which each local academic culture answers to different social and political challenges departing from their differing specific traditions and, in so doing, they achieve to play their public and critical role.

Araujo, K., 2015, "Culturas académicas: entre reinvención y contrabando", en Papeles del CEIC, vol. 2015/1, no 118, CEIC (Centro de Estudios sobre la Identidad Colectiva), Universidad del País Vasco, http://dx.doi.org/10.1387/pceic.13014 


\section{1. ¿QUÉ SIGNIFICA MIGRAR?}

Hace ya algunos años me tocó ser jurado en un concurso de cuentos para mujeres migrantes en Chile. Fui encargada en aquella ocasión de dar el discurso en la ceremonia de entrega de los premios. El contenido estricto del discurso no tiene importancia para este relato, pero sí el lugar de enunciación desde el que fue producido: lo escribí apelando a mi propia condición de migrante, una cuestión que también había estado en la base de la decisión de mis compañeros de jurado al nombrarme su portavoz. Cuando terminó la ceremonia, una colega mía se acercó y con voz comprensiva pero con un audible tono de reproche me señaló que era un error hacer la equivalencia entre su migración (la de mujeres empleadas domésticas, presas por narcotráfico o trabajadoras manuales) y la mía, la de una académica, investigadora y profesora universitaria. Además, dijo, en verdad, y más allá de la nacionalidad, piénsalo, tú eres chilena. Tenía razón. Por un lado, porque no podía de buena fe comparar experiencias que sumaban a la migración padeceres de clase, de género, en muchos casos étnicas, y de nacionalidad, con mis propios padeceres. Por otro lado, también era cierto que, en un sentido, mi condición de migrante no era un factor explicativo central de lo que me acontecía profesionalmente. Mi trabajo en Chile, desde mi llegada hace más de quince años, ha estado de manera mayoritaria asociada a investigaciones sobre el pais; he participado desde muy temprano en grupos de investigación regionales como investigadora chilena; y solo de manera extremadamente excepcional, debo reconocer, he sido puesta en cuestión en mi trabajo por el hecho de no haber nacido en el país. Para ponerlo en sus palabras, era cierto que no solo era una académica sino una académica chilena. Pero, al mismo tiempo, mi interlocutora crítica no tenía razón, y no la tenía porque había algo en común entre aquellas mujeres y yo. Estábamos aunadas por dos de las experiencias primarias que implica la migración, a saber, el empuje a la reinvención y el ejercicio del contrabando.

En efecto, aunque incomparables en términos de padeceres, sus experiencias y las mías hacían eco en algo que había podido leer entre las líneas en todos aquellos relatos: el arduo trabajo de reconstrucción de las referencias en torno a las cuales organizar sus modos de actuar en el mundo que debian enfrentar; la perplejidad ante la ineficacia de los ideales que traían; el sentimiento de encontrarse a la intemperie dada la 
escasez de experiencias acumuladas que les mostraran las lógicas actuantes en el nuevo mundo que debían habitar. Ellas, como yo, habian tenido, y tenian, de manera constante y renovada que enfrentar el cambio de las coordenadas de la orientación cotidiana de sus actos. Un trabajo, me parece, que se constituye, sin lugar a dudas, en uno de los más exigentes, estimulantes pero también opacos a los que enfrenta la migración. Los encuentros con nuevas formas de sociabilidad obligan a lecturas delicadas y cuidadosas de nuestros presupuestos para la acción. Estos encuentros, así, simultáneamente iluminan nuestras experiencias anteriores y nos empujan a un ejercicio de reinvención del que ni ellas ni yo alcanzábamos a escapar.

La segunda razón por la que mi colega no tenía razón en echar por tierra mis pretensiones identificatorias con aquellas mujeres migrantes de la escritura de los cuentos, era inversa a la anterior. Ellas, sus textos, revelaban una no siempre explícita pero siempre presente conciencia de la insistencia de sus determinaciones de origen. Esto es, que si migrar es reinvención, la migración es, al mismo tiempo, un ejercicio de contrabando. Quien migra, lo sabemos todos, trae en el cuerpo, en las palabras y en las texturas de su voz, el lugar que dejó. Son los efectos de sus apegos ideales, de las cargas afectivas que definen el grado de luz $u$ oscuridad con las que se arman las imágenes cotidianas, de los saberes sobre lo social que se han acumulado y que han ido a parar, quién sabe por qué extraño camino, a una risa demasiado explícita para las sensibilidades locales, a una distancia inadecuadamente sostenida con los otros cuerpos o a una reacción emocional ante escenas que no tocarian a ningún nativo. Se porta consigo usos, incluso tan sutiles que resultan imperceptibles para una misma, que contravienen aquellos ordinarios. Una migrante es, quiéralo o no, sea cual sea su esfuerzo por integrarse, siempre una extranjera. Lo es porque hay algo irreductible e irrenunciable en ella (o él): el monumento palpitante de sus apegos anteriores.

Para decirlo en términos de Isaiah Berlin, quizás especialmente adecuados para hablar de desplazamientos intelectuales, hay fibras de zorra y de erizo en aquellos que se desplazan. Como se sabe, Isaiah Berlin en su sensible análisis de la obra de Tolstoi ha movilizado con sabiduría las dos figuras que el poeta griego Arquíloco refiere en su famoso fragmento: "Muchas cosas sabe la zorra, pero el erizo sabe una sola importante". En su lectura, la zorra es móvil y se ocupa de muchas cosas 
a la vez, y, dada la multiplicidad de la que se nutre, su fe en las verdades únicas es reducida. El erizo, en cambio, es sistemático y está movido por una mirada coherente y totalizante del mundo. Pero, el estado puro no es lo usual, y las combinaciones son múltiples (Berlin, 2002). Hay erizos que parecen a primera vista zorras; zorras bajo la capa del erizo; zorras con corazón puro de zorras, y, claro, hay más... Por ejemplo, los de fibras mixtas, el tipo que, me parece, cabe a cada uno y todos los que han hecho del desplazamiento geográfico e intelectual más o menos permanente un rasgo de sus trayectorias singulares, por lo menos en aquellos casos en que no fueron resultado de coacciones ineludibles, como en el caso de algunos de los exilios políticos.

Detrás de las opciones de dejar el lugar de origen para dedicarse a conocer, pensar o experimentar una realidad que nos es extraña hay algo de la curiosidad por el mundo, pero también de la convicción, de zorra, acerca no solo de la pluralidad de la verdad que le concierne, como le gustaría subrayar a Berlin, sino, también, de los estilos de habitarlo. Las razones que nos damos para tomar la decisión de dejar nuestros lugares de origen siempre serán formas más o menos intelectualizadas de dar cuenta de un desapego que se impone. Más allá de las características particulares de cada biografía, siempre hay una razón de fuerza para partir, pero, también, y sobre todo, hay una pasión para insistir en una aventura cuya exigencia principal es estar abiertos a que las cosas pueden ser siempre de otra manera. Y, al mismo tiempo, quien se desplaza porta consigo sus límites. No me refiero solo y ni siquiera principalmente a las determinaciones inevitables de la personalidad o el carácter propio sino a que, en rigor, todo desplazamiento, como lo ha señalado Freud en su análisis del inconsciente y los sueños, supone de manera esencial una transferencia de las valencias de la posición original hacia la que le sigue. Cuando uno se desplaza, quiero decir, lo que está en juego no es solo la cuestión de ocupar un espacio distinto sino el hecho enorme que las catexias y los materiales vinculados a nuestros apegos originales nos acompañan en nuestra instalación en los lugares de llegada.

Es, precisamente, desde esta conjunción entre un desapego que se entrama en la creencia en la pluralidad y unos nuevos lazos que son hechos de materia que proviene de nuestros apegos originarios, que la reinvención y el contrabando pueden ser situados. En cuanto zorras estamos siempre listas para la reinvención. Como buenos erizos 
estamos atados a una singularidad que será la mercancía de contrabando con la que llegamos a nuestros lugares de acogida.

En lo que corresponde a mi itinerario personal son al menos dos los grandes desplazamientos que vertebran $\mathrm{mi}$ biografía personal $\mathrm{e}$ intelectual hasta hoy: un desplazamiento geográfico (el paso de Perú a Chile mediado por una estadía de algunos años en Alemania); y un desplazamiento disciplinario (el paso del psicoanálisis a la sociología). Todo ello, claro, en el contexto de los micro-desplazamientos constantes a los que obliga el hecho de ser una mujer en un mundo, aún, y a pesar de las grandes transformaciones, con una clara hegemonía masculina. Si bien hay muchas maneras en que podrían interrogarse estos movimientos, en este texto me gustaría abordar solo una de ellas: lo que mi desplazamiento geográfico, las experiencias en la reinvención y en el contrabando, me han mostrado acerca de la importancia y peso de las culturas académicas locales.

Dos cuestiones de fondo en mi voluntad argumentativa en este texto son: 1) recordar el valor constitutivo y extremadamente decisivo de las lógicas valóricas y de sociabilidad de las culturas académicas para cada universo intelectual y profesional, $y$, principalmente, 2) subrayar el hecho que las culturas académicas no son meramente idiosincráticas, sino que son el resultado de las exigencias que las disciplinas deben enfrentar en momentos históricos determinados contando con el conjunto de los recursos, obstáculos y aprendizajes que el acumulado de su trayecto (tradición) les aporta. Me interesa discutirlo particularmente porque, a mi juicio, las exigencias de homogeneización que la cultura de la evaluación trae (Ortiz, 2009), y los riesgos de la creciente imaginería de lo global para las ciencias sociales (Wieviorka y Calhoun, 2013), tienden a recubrir esta diversidad, esto es, la multiplicidad de las culturas académicas, y, con ello, neutralizan una reflexión más detenida sobre su importancia para garantizar la vocación pública de las ciencias sociales, y de la sociología en particular.

\section{LAS CULTURAS ACADÉMICAS LOCALES}

Para quienes han migrado, como yo, pero también para muchos que se han desplazado a medios intelectuales otros a los propios por algún tiempo, resulta claro, me parece, que existen culturas académicas locales. Por supuesto, esta percepción es principalmente relevante y 
aguzada, es de imaginar, para quienes se dedican a las ciencias sociales, un tipo de conocimiento que no hace sino estar confrontado de manera permanente con la cuestión del contexto en que se produce. Ahora bien, como con frecuencia este tipo de saber sobre las especificidades locales es sospechoso de estar a demasiada poca distancia del estereotipo, termina usualmente por ser mantenido en la intimidad, incluso a veces no confesada, y a no ser discutido públicamente sino en instancias muy informales. La sospecha misma de que estamos frente a estereotipos termina por ser performática y las alusiones a estas cuestiones acaban siendo comentarios rápidos al pasar, extremadamente generalizadores (del tipo, "los brasileños siempre tienen tiempo para atender con alguna celebración a sus invitados" o "los franceses no hacen sino hablar mal unos de otros"). Sin embargo, la cuestión de las culturas académicas no es un asunto banal. Ella merece una detención y una indagación que nos conduzca más allá de la superficie de afirmaciones que aparecen como excesivamente simples y estereotipadas. Quien ha hecho desplazamientos geográficos intelectuales, me parece, difícilmente podría tener dudas sobre este punto, y menos aún acerca de la manera en que ello afecta el propio trabajo. Más allá de la simple anécdota, una cultura académica local tiene efectos sobre los modos en que se produce, se discute o negocia, y se disemina el conocimiento.

\subsection{Los años peruanos}

Cuando llegué a Chile, tenía tras de mí ya algunos años de formación en la cultura académica peruana, tanto por mis estudios como por mis lecturas, pero, también, por la suerte de una temprana participación en el mundo académico gracias a un puesto en la universidad que gané pocos años después de haber cumplido veinte.

En el Perú de mis años formativos y del inicio de mi vida profesional había algunas cuestiones que resultaban componentes indispensables para alcanzar lo que constituía el modelo más prestigioso de la práctica en ciencias sociales en ese momento ${ }^{1}$. La primera, una familiaridad con los clásicos del pensamiento social peruano $y$, por extensión, con los

\footnotetext{
${ }^{1}$ Las culturas académicas no son entes cristalizados sino que se encuentran sujetas a transformaciones históricas. Al mismo tiempo, si bien hay modelos hegemónicos que reúnen mayor prestigio en un determinado momento, que son a los que me referirá aquí, normalmente están acompañados de otras modalidades que difieren o entran en abierta confrontación con ellos. Es con estas prevenciones que el relato siguiente debe ser considerado.
} 
debates y la producción locales. No era posible hacer parte de la comunidad de discusión sin haber pasado de manera seria por la lectura de José Carlos Mariátegui, Haya de la Torre o por la obra de Basadre, por ejemplo; sin llevar en el imaginario la mesa redonda sobre la novela Todas las sangres, y la confrontación que ella supuso entre su autor, José María Arguedas, y otros conocidos intelectuales como Salazar Bondy o Aníbal Quijano; sin tener posición respecto a las lecturas sobre el Perú más contemporáneas como aquellas de Julio Cotler, Pablo Macera, Flores Galindo o las que desde el psicoanálisis brindaba Max Hernández. Con seguridad, la fuerza concéntrica del debate peruano no se redujo a mi generación, y tiene una larga historia, pero también es posible que esta exigencia fuera aguzada por el momento histórico que atravesábamos. Mi generación fue testigo de la debacle política de la izquierda tradicional, $y$, al mismo tiempo, enfrentó sin muchos recursos porque quizás demasiado joven o porque demasiado asustada, el desgarro que constituyó para el país la lucha armada de Sendero Luminoso y el Movimiento Revolucionario Túpac Amaru (MRTA) y la respuesta represiva del aparato estatal. La urgencia por las respuestas y los recursos limitados ante una realidad que sobrepasaba cualquier imaginación se combinaron para generar un ambiente de desconcierto $y$, al mismo tiempo, sediento de grandes respuestas, al menos en los primeros años.

En este contexto, constituido por la tradición de pensamiento nacional y las exigencias de un momento histórico dramático, las grandes interpretaciones sobre el país asentadas en una lectura de su trayectoria histórica de largo plazo eran el telón de fondo permanente desde el que resultaba deseable, posible y consistente realizar cualquier acercamiento o contribución analítica de peso. La tradición de pensamiento peruano hacía que jóvenes aspirantes al trabajo intelectual, como yo en aquel momento, no solo se sintieran obligados a una reflexión muy anclada en los aportes locales (aunque, paralelamente me dedicara con ahínco y hasta testarudez a la lectura de las obras completas de ¡Jacques Lacan!), sino, también, que el trayecto se nutriera con la convicción que esas interpretaciones globales no solo eran necesarias sino posibles. No había por qué dudar de la plausibilidad de esas grandes interpretaciones que daban la clave para entender el país, pero era obvio que había que trabajar duro para alcanzar la 
erudición que una tal empresa requería y echar mano de todo aquello que se tuviera a mano en la tarea.

Erudición, quería decir, en este caso, no solamente una profundización sistemática y abarcadora sobre la temática, es decir, el "trabajo del erizo", volviendo a Berlin, que sabe todo de aquello que es su objeto de conocimiento, sino, también, a otra modalidad de la erudición: aquella que no respeta fronteras. Una interpretación global se asociaba con frecuencia a una enorme legitimidad en el uso de recursos que atravesaban las fronteras disciplinarias. Nuestras lecturas de los que considerábamos entonces nuestros maestros (una figura cuyo uso puede entenderse si se toma en cuenta la densidad de la tradición de pensamiento local), nos mostraban que acudir a otras disciplinas no solo era admitido sino inmensamente valorado. De este modo, parte de nuestro arsenal estaba compuesto por libros, por ejemplo, que hacian del Inca Garcilaso de la Vega metáfora nacional y lo estudiaban desde el psicoanálisis y la historia (Max Hernández, 1993); o de momentos míticos, como el de la mesa redonda sobre la novela Todas las Sangres en los años sesenta caracterizado por el encuentro explosivo entre novela y ciencias sociales en la disputa por la interpretación del país (Rochabrun, 2011), solo por nombrar algunos casos. La exigencia a la lectura era redoblada, es cierto, pero al mismo tiempo, y esto no es menor, se justificaba una buena cuota de libertad experimental, la que resultaba celebrada por el medio.

Por otro lado, en un país afectado por una creciente crisis de legitimidad del sistema político y en el que el Estado era parte de los fenómenos transversales de corrupción y violencia, el pensamiento crítico, y, por tanto, las tareas de las ciencias sociales, debian, necesariamente, tomar distancia de toda alianza con él. La autonomía, la figura del pensador crítico comprometido y por tanto ubicado en la vereda opuesta del aparato gubernamental ${ }^{2}$, nutría de manera significativa el modelo del intelectual.

Una última cuestión, que en verdad hay que colocarla en el contexto de los rasgos más generales del país, tiene que ver con el uso del lenguaje. En cualquier sociedad hay elementos distinguidos que sirven como

2 No hay que olvidar que lo gubernamental en aquellos años refería a figuras presidenciales como Alan García o Alberto Fujimori, ambos responsabilizados por matanzas y sospechosos de uso indebido de los fondos estatales. 
marcadores diferenciales de las posiciones ocupadas en el mundo social (Bourdieu, 2010). Un elemento de distinción muy importante en el caso del Perú, por mucho tiempo, fue el buen y rico uso de la lengua castellana. Riqueza lexical, el uso de conjugaciones verbales complejas, la propiedad gramatical, el uso de ciertas fórmulas retóricas y una pronunciación pulcra y sin demasiada prisa ${ }^{3}$, constituyeron un arma para la distinción de las élites económicas, los sectores educados, y de los grupos medios (Parker, 1998), unos respectos de otros pero también de éstos respecto del contingente de grupos indígenas en zonas provinciales o de los migrantes internos, quechua o aymara hablantes especialmente, incluso de segunda generación, con un reducido o específico manejo de la lengua castellana. Por mucho tiempo, la capacidad retórica, la habilidad evocativa y la destreza descriptiva resultaron cuestiones centrales que definían lo que podía ser considerado valioso intelectualmente, y aunque para mi generación este ideal comenzaba a perder nitidez enfrentado a convulsionados procesos sociales y políticos que hacían emerger nuevos modelos, no cesó de ejercer su impronta.

En breve, mi experiencia me mostraba que el mundo intelectual y la producción en ciencias sociales debía nutrirse extensamente de la tradición del pensamiento social peruano y de su producción, que era un deber conocerla; que la cúspide de este tipo de contribuciones se asociaba con la generación de interpretaciones generales del país con base en su historia de largo plazo; que la interdisciplinareidad, fuente de la experimentación analítica e interpretativa, era una herramienta central en esta empresa; que el pensamiento crítico suponía una clara sospecha respecto al Estado y al sistema político. Por último, y englobando todo lo anterior, que se requería de la presencia de un amor muy dedicado por el lenguaje y de refinamiento retórico, ambas habilidades indispensables para cumplir con la tarea propuesta.

\subsection{Singularidades chilenas}

Llegué a Chile a mediados de los noventa. Chile, como había empezado a hacerlo Alemania en los años en los que viví y estudié en ese país, dio nitidez al carácter del saber que provenía de mi experiencia peruana, pero, al mismo tiempo, me empujó a revisarlo. Muy temprano resultó

3 Un ejemplo prístino y destacado de esta figura extendida en el mundo intelectual peruano es, sin duda, el escritor Mario Vargas Llosa. 
evidente que ese saber no me permitiría participar en las conversaciones académicas en el nuevo entorno sin el riesgo de producir disonancias tan grandes que impidieran la comunicación.

En el momento histórico que me tocó participar en el Perú, el modelo hegemónico de prestigio para las ciencias sociales y la discusión mantenían una fuerte personalidad intelectual de raíces históricas pero estaban especialmente tocadas por un momento de crisis y violencia, lo que desembocaba progresivamente en pesimismo y desconfianza acerca de los destinos del país y de su posible contribución apoyando el papel del Estado. A mi llegada a Chile, por el contrario, a seis años de la vuelta a la democracia, las ciencias sociales retornaban a la universidad y daban sus primeros pasos en ella luego de su exclusión ( $y / 0$ debilitamiento) de estas instituciones por parte de la dictadura militar (Joignant y Navia, 2013), y de un desarrollo que tuvo, mientras que duró aquella, como escenario privilegiado organismos no gubernamentales o centros de investigación independientes. Al mismo tiempo, y en continuidad con el carácter político que las distinguió bajo la dictadura, las ciencias sociales se encontraban fuertemente comprometidas en el apoyo a las tareas de reconstrucción o remodelación democrática que se impusieron los cuatro gobiernos de la Concertación (1990-2010). Lo anterior, exigía un alto grado de profesionalismo técnico. Por otro lado, dada la acendrada política consensual que caracterizó estos gobiernos, en contra de la vocación de oposición desarrollada en los años anteriores a la vuelta democrática, esta nueva situación les exigía moderación crítica ${ }^{4}$.

De este modo, y como es evidente, el campo de las ciencias sociales al que llegué, y quizás es justo decirlo, en particular el de la sociología, dadas las tareas históricas que se imponía, exigía para participar en él cosas diferentes a las que estaba habituada.

Punto por punto los contenidos de mis ideales y lo que mis experiencias me habian mostrado sobre la tarea del trabajador de las ciencias sociales, fueron puestos en cuestión.

En primer lugar, apareció la sorpresa por la inexistencia de la reverencia a la tradición del pensamiento social que yo había conocido. El mundo

\footnotetext{
${ }^{4}$ También aquí, los rasgos del campo han sufrido transformaciones a lo largo del tiempo. Por razones de espacio no los puedo detallar aqui detenidamente. Para una mirada longitudinal hasta la década de los noventa ver: Garretón, 2005.
} 
estaba todo por hacerse por lo que habia que orientarse principalmente a herramientas teóricas que fueran más actuales que venerables. Esto no quiere decir, por cierto, que la impronta de la obra de sus historiadores o intelectuales de la era republicana no fueran relevantes como tampoco que no existieran discusiones acerca de algunas colaboraciones y producciones de sus científicos sociales más contemporáneos. Pero, al menos desde el momento de mi llegada, lo que se ha ido agudizando con el paso del tiempo y las exigencias de la evaluación y los indicadores que utiliza, un elemento extremadamente importante para la producción de conocimientos resultaba la familiaridad con la producción intelectual extranjera, particularmente del norte. Quizás vale la pena aclarar este punto.

No es mi intención ni de lejos proponer que en el Perú no se trabajara autores europeos o norteamericanos, como tampoco que en Chile no se leyera o discutieran las obras de historiadores como Góngora o Gabriel Salazar, por ejemplo. Lo que me interesa subrayar es que la densidad libidinal del conjunto de autores locales para la conformación del campo de discusiones resultaba diferencial en cada uno de los casos. Estas distinciones se revelan, por ejemplo, en la importancia dada a sus intelectuales o en el peso de la historia de largo plazo en cada una de estas culturas académicas, al menos en los momentos de mi encuentro personal con ellas. Si en el caso del Perú, una mirada histórica de muy largo plazo era el nutriente principal exigido a toda reflexión considerada sustantiva en ciencias sociales, en el caso de Chile, la historia de largo plazo y las diferentes interpretaciones del país no despertaban ni las mismas pasiones ni jugaban el mismo papel o solo lo hacian en algunas importantes excepciones. Un síntoma de lo anterior es que no se encontraba, ni se encuentra, en esta cultura académica un equivalente de la generalizada actitud admirada e incluso reverencial que podían y pueden aún suscitar figuras del pensamiento social (y por su contribución a éste) como la de José Carlos Mariátegui o la de José María Arguedas en el Perú.

En segundo lugar, en mi encuentro con la cultura académica chilena, y en mucho, seguramente, debido a los cristales comparativos con que debí descifrarla, un elemento resaltante era la limitada legitimidad de esfuerzos de interpretación global de gran envergadura acerca del país que incluyeran su trayecto histórico de largo aliento. Lo que aparecía como el horizonte y referente histórico principal, en este caso, era la 
dictadura, lo que implicaba un límite en los años setenta del siglo XX. Este hecho se asociaba, es solo una de las razones posibles, con que ella era leída como una cesura que rompía con la continuidad de la historia del país e iniciaba un ciclo nuevo. Dicho de otro modo, las lecturas históricas de largo aliento no resultaban demasiado útiles porque la dictadura fue interpretada mayoritariamente como un momento de excepcionalidad en el trayecto nacional, pero a la que se daba la más gravitante importancia pues contenía las claves para entender el momento actual.

Un buen ejemplo es uno de los momentos más fructíferos y hasta excepcionales del debate intelectual chileno: la discusión sobre modernidad y modernización, que se estableció en la década de los noventa (Brunner, 1994; Larraín, 1996; Moulian, 1997; Bengoa, 1996 Jocelyn-Holt, 1997, PNUD; 1998), el que se desarrolló en torno a la confrontación entre posiciones críticas y otras más optimistas sobre el futuro y el significado de los procesos de modernización, ligados a la instalación del modelo neoliberal en la sociedad (Pinedo, 2000). A pesar de la profundidad de la discusión, y las referencias que se hicieron a la historia, no implicó, excepto en muy pocos casos, movilizar lecturas históricas de largo aliento que pusieran en el centro la tradición de posiciones en disputa por la interpretación del país. De otro lado, el hito referencial histórico explicativo del momento actual principal era la dictadura o, como mucho, pero solo en relación directa con la dictadura, lo que se consideró su directa referencia, la figura de Portales en el siglo XIX. Finalmente, elementos que se movilizaron a nivel argumentativo fueron, de manera importante, datos contemporáneos de diagnóstico sobre diferentes aspectos de la sociedad chilena, como tasas de consumo, conformidad, confianza o indicadores de malestar.

Otra línea explicativa para la falta de centralidad y prestigio de estas explicaciones históricas de largo aliento es que, dadas las tareas de apoyo al Estado en democracia, la producción se encontraba, aunque no completamente, por cierto, sometida a la implícita demanda de dar cuenta de la aplicabilidad o del sentido práctico más inmediato de los resultados obtenidos, algo de lo que difícilmente sería capaz una teoría general de explicación de la sociedad. Como contrapartida se imponía un mayor prestigio de estudios focalizados en temáticas específicas. La creciente hegemonía, en aquel momento y por largo tiempo, de lo que Burawoy llama policy sociology (2004), reemplazó, así, una práctica 
crítica particularmente importante en la época de la dictadura y en la lucha contra ella. Cuestiones como estudios sobre políticas públicas, eficacia de políticas sociales o modernización del estado, resultaban especialmente relevantes. La colonización de las agendas de la sociología por la agenda estatal y, de manera más general, por la esfera política institucional (Mascareño, 2010), se vinculó fuertemente con el retorno de la democracia y con las lealtades (pero también clientelismos) que este proceso produjo. La tarea de aportar a construir otro estado, reestructurar sus relaciones con la sociedad y de apoyarlo en sus funciones, resultó especialmente gravitante para definir el compromiso básico de muchos científicos sociales tras diecisiete años de dictadura.

La tercera diferencia con la que me topé, a cuenta de mi desplazamiento geográfico, se relaciona con la importancia de las cuestiones metodológicas. Mientras las interpretaciones globales prestigiosas en el caso peruano, ponían por encima la imaginación al método, a veces de maneras extremadamente disyuntivas, éste no era el caso en el medio chileno. En efecto, un o una practicante enfrentaba un control mayor sobre los mecanismos y procedimientos de la producción de conocimientos. Es decir, tenía que observar un peso y una vigilancia más estricta de las cuestiones técnicas y metodológicas, así como, también, de los límites interpretativos y los grados de generalización de los conocimientos producidos. En consecuencia, y como es evidente, el pluralismo y la audacia teórica y metodológica, tan celebrada en mi época formativa y de inicio profesional peruano, concitaban más curiosidad que entusiasmo. Lo que podía ser considerado como una contribución relevante debía someterse a claras exigencias de rigor, muchas veces disciplinario, y, por tanto, contaba con un espacio sensiblemente menor para los emprendimientos interdisciplinarios y las búsquedas experimentales.

Finalmente, y tomando en cuenta lo anterior, resulta explicado que las formas de comunicación de los resultados científicos exigieran otro uso del lenguaje. La retórica y el trabajo evocativo propios de las grandes interpretaciones globales quedaban reemplazados por un lenguaje más directo y denotativo. El ejercicio de la retórica y la evocación, por el contrario, resultaban sospechosos. Por supuesto, aquí, me parece, también es posible ensayar una hipótesis más general de tipo lingüística y psico-social, respecto a cómo esta distancia pudiera reflejar una cierta 
desafección que se vincularía con la tendencia de los chilenos en su percepción lingüística al español chileno como uno de los más incorrectos de los paises hispanohablantes (Rojas, 2012), pero creo que no es tan claro que vaya principalmente en esta dirección. Estos rasgos del uso lingüístico se encuentran ligados con las exigencias del tipo de conocimiento que debía ser producido en las ciencias sociales, que privilegiaban la transmisibilidad y la claridad. En la reconstrucción que puedo hacer desde hoy, es posible que este desencaje haya sido la razón por la cual a pesar de que tempranamente empezó mi actividad de investigación en ciencias sociales, por un buen tiempo mis interlocutores favoritos, aunque nunca cupiera demasiado bien yo allí, fueran aquellos que venían de los estudios culturales, particularmente de la literatura, la filosofía y las artes plásticas.

\subsection{Decantados en saber}

Describir en detalle la manera en que se resolvió en mi caso esta reinvención y cuáles mis contrabandos al mundo académico que me acogió, me parece, no es demasiado interesante para los fines de una reflexión como la presente. $Y$, además, mi esfuerzo sería vano a falta de la distancia necesaria. Por supuesto, lo que es seguro es que mi oficio de socióloga no lo habría desarrollado de la misma manera si no hubiera habido este desplazamiento entre culturas académicas. Seguramente, si no hubiera sido por mi desconfianza heredada de los años peruanos, no habría resistido tan activamente a que mis agendas de investigación fueran moldeadas por la relación con el Estado; puede ser que si no hubiera sido por el desprestigio que tenían en la cultura académica chilena, habría intentado en mis trabajos interpretaciones más arriesgadas y globales; $y$, tal vez, si no me hubiera encontrado con las exigencias con las que me encontré habría estado menos preocupada metodológicamente en el desarrollo de mis investigaciones. Quién sabe. En cualquier caso, lo que es innegable es que me vi confrontada, como todo aquel que atraviesa la experiencia migratoria, a realizar un ajuste inventivo, una reinvención que me permitiera orientarme con pies más firmes en el mundo que empecé a habitar, pero que estaba destinada a no poder sustraerse del todo de mis apegos originarios, de los cuales, quizás, mi relación con el lenguaje sea la más evidente.

Pero, lo que sí resulta esencial, a mi entender, es el saber que se decantó desde una experiencia como ésta, y ello a pesar de mis resistencias 
iniciales y los no pocos esfuerzos que me exigió y reclamé: esto es, que no había una cultura académica mejor que otra. Que no se trataba de comparar sino de entender. Lo que yo encontré en aquellos dos momentos de mi vida fueron conjuntos de principios ideales $y$ exigencias prácticas para quienes pretendíamos hacer parte de los trabajadores de las ciencias sociales, y, más allá de mi simpatía mayor o menor por algunos de sus componentes, estos conjuntos no eran arbitrarios sino que tenían profundas y complejas lógicas propias. En ambos casos, se trataba de ciencias sociales comprometidas socialmente, con formas particulares de entender las tareas históricas a las que ellas estaban enfrentadas, y con herencias y trayectos diferenciales que influyeron en las modalidades en que les resultó posible responder a estos requerimientos. Con sus éxitos y fracasos, con sus excesos y sus límites, con todo lo que se les pueda criticar o añorar de cara al momento actual, cada una de ellas, en cada uno de esos universos sociales circunscritos a un momento histórico, intentaba responder, y esto es esencial, a su carácter público.

De este modo, por contraposición, mi historia de desplazamiento, su empuje a la reinvención y al contrabando, muestra de qué manera las modalidades de evaluación hoy, los rankings, la búsqueda de isomorfismo de las estructuras argumentativas y las formas lingüisticas o incluso la ficción de sociologías globales, son los hilos de un manto (de los muchos que nuestra época de capitalismo avanzado tiende a producir) que recubre la importancia y la vigencia política, en el sentido más amplio del término, de mantener vivas las culturas académicas particulares. El carácter público, crítico y comprometido de las ciencias sociales, y en particular de la sociología, está indefectiblemente unido a la fidelidad a las tareas específicas, muchas veces urgentes, que las realidades en que se desarrollan les plantean. A la conciencia que esos son los desafíos que les competen. Esto quiere decir que las divergencias en las culturas académicas, la singularidad de sus estilos, las particularidades valóricas, las formas de sociabilidad que las distinguen y hasta sus formas lingüisticas son una, cierto que entre otras, garantía de que la sociología pueda quedar aún leal a la vocación que la atinge desde su origen.

Si no conseguimos hacer algo contra la expansión de los procesos homogeneizadores, un día en el futuro algún estudiante que se anime a desempolvar viejas historias se preguntará, con sorna y hasta con 
lástima, cómo una comunidad que fue capaz de desarrollar vibrantes evidencias y alegatos acerca de la importancia de la diferencia; que se desarrolló en un momento de quiebre epistemológico en el que los paradigmas únicos resultaban insostenibles; que fue la hija del giro lingüistico; y que estuvo alimentada por tanta experiencia de migración, permitió y hasta se auto-impuso entusiastamente procesos tan extendidos de uniformización. Tendrá razón.

\section{BIBLIOGRAFÍA}

Bengoa, J., 2006, La comunidad perdida. Ensayos sobre identidad y cultura: los desafíos de la modernización en Chile, Sur, Santiago.

Berlin, I., 2002, El erizo y la zorra, Península, Barcelona.

Bourdieu, P., 2010, La distinción. Criterios y bases sociales del gusto, Taurus, Madrid.

Brunner, J. J., 1994, Bienvenidos a la modernidad, Planeta, Santiago.

Burawoy, M., 2004, "Public Sociologies: Contradictions, Dilemmas, and Possibilities", en Social Forces, 82(4), pp. 16-18.

Garretón, M. A., 2005, "Social sciences and society in Chile: institutionalization, breakdown and rebirth", en Social Science Information, Vol. 44(2 \& 3), pp. 359-409.

Hernández, M., 1993, Memoria del bien perdido: conflicto, identidad y nostalgia en el Inca Garcilado de la Vega, IEP/Biblioteca Peruana de Psicoanálisis, Lima.

Jocelyn-Holt, A., 1997, El Peso de la Noche. Nuestra frágil fortaleza histórica, Ariel, Buenos Aires.

Joignant, A., P. Navia, (Comps.), 2013, Ecos mundiales del golpe de Estado. Escritos sobre el 11 de septiembre de 1973, Ediciones Universidad Diego Portales, Santiago.

Larraín, J., 1996, Modernidad. Razón e identidad en América latina, Andrés Bello, Santiago.

Mascareño, A., 2010, Diferenciación y contingencia en América Latina, Universidad Alberto Hurtado, Santiago.

Moulián, T., 1997, Chile actual. Anatomía de un mito, Lom-Arcis, Santiago.

Ortiz, R., 2009, La supremacía del inglés en las ciencias sociales, Siglo XXI, Buenos Aires. 
Parker, D., 1998, The Idea of the Middle Class: White-Collar Workers and Peruvian Society, 1900-1950, The Pennsylvania University Press, University Park, Pennsylvania.

Pinedo, J., 2000, "Ensayo chileno y política: algunas propuestas de fin de siglo", en R. Cánovas y R. Hozven (Eds.), 2000, Crisis, Apocalipsis y Utopias, Ed. PUC, Santiago, pp. 429-435.

PNUD (1998), Informe de Desarrollo Humano en Chile. Las paradojas de la modernización, PNUD, Santiago.

Rochabrun, G., (Ed.), 2011, “¿He vivido en vano?" La mesa redonda sobre todas las sangres del 23 de junio de 1965, Instituto de Estudios Peruanos, Lima.

Rojas, D., 2012, "Corrección idiomática atribuida al español de los países hispanohablantes por sujetos de Santiago de Chile", en Revista de Lingüística Teórica y Aplicada, 50 (2), II Sem., pp. 39-62.

Wieviorka, M., C. Calhoun, 2013, "Manifeste pour les Sciences Sociales", en Socio, $n^{\circ} 1$, mars, pp. 3-38. 